\title{
Performances Zootechniques des Elevages de Dindon Local (Meleagris Gallopavo) dans la Commune de Ouaké au Nord-Ouest du Bénin
}

Vidjinnangni Fifamè Grâce Nadège Dèdéhou, Senior Lecturer-Researcher, Laboratoire d'Ecologie, Santé et Production Animale, Faculté d'Agronomie, Université de Parakou, Benin Laboratoire d'Ethnopharmacologie et de Santé Animale, Faculté des Sciences Agronomiques, Université d'Abomey-Calavi,Cotonou, Benin

\author{
Ibrahim Alkoiret Traoré, Professor \\ Attakpa Yatchégnon Eloi, Associate Professor \\ Laboratoire d'Ecologie, Santé et Production Animale, \\ Faculté d'Agronomie, Université de Parakou, Bénin
}

Doi:10.19044/esj.2019.v15n36p58～URL:http://dx.doi.org/10.19044/esj.2019.v15n36p58

\section{Résumé}

Suite à une enquête zootechnique qui a eu pour objectif d'établir la typologie des élevages de dindon de la commune de Ouaké, les performances de croissance et de reproduction desdites exploitations ont été étudiées. Les résultats montrent que le poids, les paramètres de reproduction ont été significativement influencés par le groupe d'éleveurs et le phénotype des dindons $(\mathrm{p}<0,05)$. Ainsi, de l'éclosion à 3 mois d'âge, les dindonneaux du groupe 3 sont plus lourds, suivis de ceux du groupe 2 et de ceux du groupe 1 $(\mathrm{p}<0,05)$. Les dindonneaux bronzés sont plus lourds $(\mathrm{p}<0,05)$ que les dindonneaux noirs et gris. A 6 mois d'âge, le groupe et le phénotype ont influencé significativement le poids des dindons. Les femelles du groupe 3 ont eu un poids vif supérieur $(p<0,05)$ à celui des deux autres groupes et les dindes bronzées sont plus lourdes que les noires et les grises. Les dindes du groupe 3 ont eu de meilleures performances de ponte et de reproduction que celles des groupes 2 et $1(\mathrm{p}<0,05)$. Les taux d'éclosion ont été statistiquement différents et ont été de $55,4 \%, 65,7 \%$ et $70,5 \%$ respectivement pour les groupes 1,2 et 3 . Les meilleures performances zootechniques ont donc été obtenues avec le groupe 3 et les dindons bronzés.

Mots-clés : Dindon, Croissance, Reproduction, Groupe, Phénotype, Bénin 


\title{
Zootechnical Performance of Local Turkey (Meleagris Gallopavo) Breedings in the Commune of Ouaké in Northwestern Benin
}

Vidjinnangni Fifamè Grâce Nadège Dèdéhou, Senior Lecturer-Researcher, Laboratoire d'Ecologie, Santé et Production Animale, Faculté d'Agronomie, Université de Parakou, Benin Laboratoire d'Ethnopharmacologie et de Santé Animale, Faculté des Sciences Agronomiques, Université d'Abomey-Calavi,Cotonou, Benin

\author{
Ibrahim Alkoiret Traoré, Professor \\ Attakpa Yatchégnon Eloi, Associate Professor \\ Laboratoire d'Ecologie, Santé et Production Animale, \\ Faculté d'Agronomie, Université de Parakou, Bénin
}

\begin{abstract}
Following a zootechnical survey which established the typology of turkey rearing systems in the commune of Ouaké, the growth and reproduction performances of turkey were studied. The results show a significant influence of the farmers' group and the phenotype of turkeys on the weight and reproductive parameters $(\mathrm{p}<0.05)$. Thus, from hatching to 3 months of age, group 3 turkeys were the heaviest, followed by those of group 2 and group 1 turkeys successively $(p<0.05)$. Bronzed turkeys were heavier $(p<0.05)$ than black and grey turkeys. At 6 months of age, the group and phenotype significantly influenced the weights of both sexes. The body weight of female turkeys of Group 3 was higher than the other two groups ( $p<0.05)$ when bronzed turkeys were heavier than black and grey ones. Group 3 turkeys showed better egg-laying and reproductive performance compared to the other two groups $(\mathrm{p}<0.05)$ while group 2 turkeys performed better than those of group 1. Hatching rates were statistically different and averaged $55.4 \%, 65.7 \%$ and $70.5 \%$ respectively in groups 1,2 and 3 . The best zootechnical performances were therefore obtained with group 3 and bronzed turkeys.
\end{abstract}

Keywords: Turkey, Growth, Reproduction, Group, Phenotype, Bénin

\section{Introduction}

L'aviculture au Bénin occupe une place importante dans les moyens d'existence des populations surtout en milieu rural où les volailles sont élevées non seulement pour la consommation locale, les pratiques rituelles, 
coutumières, religieuses et sociales, mais aussi pour la génération de revenus (MAEP, 2014). Elle est pratiquée par plus de $80 \%$ des populations pour la plupart rurales (Fanou, 2006) et représente la part la plus importante des effectifs du cheptel béninois avec 20120500 têtes de volaille (BCEAO, 2017). Du fait de ses nombreuses potentialités (espèce à cycle court, production plus facile et nécessitant peu d'investissements, accessible à toutes les couches de la population), l'aviculture occupe actuellement une place de choix dans les stratégies de développement et de lutte contre la pauvreté dans la plupart des pays en développement (Sonaiya et Swan, 2004). Parmi les espèces de volaille les plus élevées au Bénin, les poulets viennent en tête et sont suivis par le canard, le dindon et les pigeons. Le dindon se compare avantageusement au poulet sur deux plans à savoir la composition de sa viande et sa valeur marchande. En effet, la viande de dinde a une teneur élevée en protéines, en vitamines et en minéraux (Ribarski et al., 2001). La dinde est considérée comme une viande ayant une faible teneur en matières grasses et en acides gras saturés, ces composés étant associés à l'apparition des maladies cardiovasculaires (Acsherio et al ., 1994; Baggio et al., 2002). Elle présente donc un profil d'acides gras intéressant. La viande de dinde est un aliment peu allergénique, ce qui fait d'elle l'une des premières viandes introduites dans l'alimentation des enfants (Alekseev et al., 2005). En ce qui concerne le volet économique, le dindon à une valeur marchande plus importante que tous les autres oiseaux de la basse-cour (MAEP, 2014).

La commune de Ouaké est l'une des plus réputée au Bénin dans l'élevage de dindon (MAEP, 2014). La typologie des élevages de dindon local dans cette commune, établie par Dèdéhou et al. (2018) a permis d'identifier et de caractériser trois groupes.

L’objectif du présent travail consiste à évaluer les performances zootechniques du dindon local élevé dans les différents groupes d'élevage de la commune de Ouaké.

\section{Materiel Et Methodes}

Une précédente enquête exploratoire a permis d'établir la typologie des élevages traditionnels de dindon local de la commune de Ouaké (Dèdéhou et al., 2018). Ainsi 3 groupes d'éleveurs ont été identifiés. Le groupe 1 est constitué par les paysans. Le groupe 2 regroupe les éleveurs-paysans. Le groupe 3 correspond aux des agro-éleveurs. L'échantillon a été constitué par 10 élevages de dindon de chaque groupe, choisis de façon aléatoire. Les paramètres suivants ont été déterminés :

\section{Détermination des performances pondérales des dindons}

Pour déterminer les performances pondérales des dindonneaux pendant la phase de démarrage ( 0 à 30 jours), les poids de tous les dindonneaux 
nés pendant la période d'étude ont été relevés à $0,7,15,23$ et 30 jours après éclosion. Les dindonneaux d'une même couvée ont été identifiés par des ficelles de couleurs différentes et leur poids a été relevé à l'aide d'une balance électronique de portée $1000 \pm 1 \mathrm{~g}$. Les phénotypes (couleur du plumage) des dindes ont été aussi relevés.

Tous les animaux ayant 3 et 6 mois et les dindons adultes ont été pesés à l'aide d'un peson de $20 \pm 0,05 \mathrm{~kg}$.

Ces données ont servi à calculer les gains moyens quotidiens (GMQ) des animaux en utilisant la formule de Lhoste et al. (1993).

$$
\mathbf{G M Q}=(\mathbf{W f}-\mathbf{W i}) / \Delta \mathbf{T}
$$

$\mathrm{GMQ}=$ gain moyen quotidien en $\mathrm{g} / \mathrm{j} ; \mathrm{Wf}=$ poids final en $\mathrm{g} ; \mathrm{Wi}=$ poids initial en $g ; \Delta \mathrm{T}=$ durée entre deux pesées en jours.

\section{Détermination des performances de ponte et de reproduction des dindes}

Pour la détermination des performances de ponte et de reproduction des dindons, les éleveurs ont été soumis à un questionnaire. Les données recueillies ont permis de calculer l'âge de la première ponte, le nombre de couvées par an, le nombre d'œufs incubés, le nombre de dindonneaux éclos. Le poids des œufs a été déterminé à l'aide d'une balance électronique de portée $1000 \pm 1 \mathrm{~g}$. Certaines données telles que le nombre d'œufs pondus par couvée, le nombre d'œufs incubés, le nombre de dindonneaux éclos ont été relevées sur place au cours de l'enquête.

Ces données ont permis de calculer le taux d'éclosion suivant la formule cidessous :

Taux d'éclosion $=($ Nombre d'œufs éclos $/$ nombre d'œufs incubés $) * 100$

\section{Analyse statistique des données}

Les données collectées ont été analysées à l'aide du logiciel SAS ${ }^{\circledR}$ (Statistical Analysis System, 1989).

La procédure PROC GLM a permis d'analyser les données sur les performances individuelles de croissance et le poids vif des animaux. Les effets du groupe (1,2 et 3 ) et du phénotype (bronzé, noir et gris), ainsi que leur effet conjugué ont été déterminés.

Les procédures PROC MEANS et PROC ANOVA ont été utilisées pour les données sur les performances individuelles de ponte et de reproduction des dindons et les données sur la composition du cheptel des élevages de dindon. La structuration des moyennes a été effectuée avec le test LSD de Fisher. Les résultats sont présentés sous formes de moyennes \pm écart type. 


\section{Resultats}

\section{Performances de croissance pondérale des dindons}

\section{Croissance pondérale des dindonneaux de 0 à 30 jours après éclosion.}

La croissance pondérale des dindonneaux pendant la phase de démarrage a été suivie par des pesées hebdomadaires. Les effets du groupe et du phénotype sur les poids vifs et les GMQ des dindonneaux sont présentés dans les tableaux 1 et 2 .

Tableau 1. Moyenne des moindres carrés et écart-types des poids ( $\mathrm{g}$ ) des dindonneaux suivant les différents stades d'évolution après éclosion

\begin{tabular}{|c|c|c|c|c|c|c|c|c|c|c|c|c|c|c|c|}
\hline Variables & $\mathbf{n}$ & $\begin{array}{l}\text { P1 } \\
\text { Moy }\end{array}$ & E.T. & $\mathbf{n}$ & $\begin{array}{l}\text { P7 } \\
\text { Moy }\end{array}$ & E.T. & $\mathbf{n}$ & $\begin{array}{l}\text { P15 } \\
\text { Moy }\end{array}$ & E.T. & $\mathbf{n}$ & $\begin{array}{l}\text { P23 } \\
\text { Moye }\end{array}$ & E.T. & $\mathbf{n}$ & $\begin{array}{l}\text { P30 } \\
\text { Moy }\end{array}$ & E.T. \\
\hline $\begin{array}{l}\text { Groupe }(\mathrm{G}) \\
\text { G1 } \\
\text { G2 } \\
\text { G3 }\end{array}$ & $\begin{array}{l}25 \\
23 \\
18\end{array}$ & $\begin{array}{l}49,0 \mathrm{a} \\
53,2 \mathrm{~b} \\
56,7 \mathrm{c}\end{array}$ & $\begin{array}{l}2,8 \\
2,9 \\
2,9\end{array}$ & $\begin{array}{l}21 \\
21 \\
17\end{array}$ & $\begin{array}{l}71,2 \mathrm{a} \\
78,3 \mathrm{~b} \\
85,2 \mathrm{c}\end{array}$ & $\begin{array}{l}4,0 \\
4,2 \\
4,3\end{array}$ & $\begin{array}{l}20 \\
20 \\
17\end{array}$ & $\begin{array}{l}87,3 \mathrm{a} \\
97,9 \mathrm{~b} \\
107,6 \mathrm{c}\end{array}$ & $\begin{array}{l}5,2 \\
5,3 \\
6,1\end{array}$ & $\begin{array}{l}20 \\
19 \\
17\end{array}$ & $\begin{array}{l}102,3 \mathrm{a} \\
115,5 \mathrm{~b} \\
129,4 \mathrm{c}\end{array}$ & $\begin{array}{l}5,7 \\
6,5 \\
6,9\end{array}$ & $\begin{array}{l}20 \\
19 \\
17\end{array}$ & $\begin{array}{l}117,4 \mathrm{a} \\
134,0 \mathrm{~b} \\
150,8 \mathrm{c}\end{array}$ & $\begin{array}{l}7,1 \\
7,7 \\
8,6\end{array}$ \\
\hline $\begin{array}{l}\text { Phénotype } \\
\text { Bronzé (B) } \\
\text { Noir (N) } \\
\text { Gris (Gr) }\end{array}$ & $\begin{array}{l}36 \\
17 \\
13\end{array}$ & $\begin{array}{l}54,7 \mathrm{~b} \\
50,2 \mathrm{a} \\
49,8 \mathrm{a}\end{array}$ & $\begin{array}{l}3,6 \\
3,5 \\
3,4\end{array}$ & $\begin{array}{l}31 \\
16 \\
12\end{array}$ & $\begin{array}{l}80,5 \mathrm{~b} \\
74,1 \mathrm{a} \\
73,5 \mathrm{a}\end{array}$ & $\begin{array}{l}6,4 \\
6,2 \\
6,1\end{array}$ & $\begin{array}{l}30 \\
15 \\
12\end{array}$ & $\begin{array}{l}100,4 b \\
91,9 a \\
91,8 a\end{array}$ & $\begin{array}{l}9,1 \\
8,6 \\
8,8\end{array}$ & $\begin{array}{l}30 \\
15 \\
11\end{array}$ & $\begin{array}{l}118,7 \mathrm{~b} \\
109,0 \mathrm{a} \\
109,1 \mathrm{a}\end{array}$ & $\begin{array}{l}12,2 \\
11,4 \\
11,2\end{array}$ & $\begin{array}{l}30 \\
15 \\
11\end{array}$ & $\begin{array}{l}137,6 \mathrm{~b} \\
126,3 \mathrm{a} \\
125,4 \mathrm{a}\end{array}$ & $\begin{array}{l}15,0 \\
13,5 \\
14,3\end{array}$ \\
\hline $\begin{array}{l}\text { G*Phénotype } \\
\text { G1-B } \\
\text { G1-N } \\
\text { G1-Gr } \\
\text { G2-B } \\
\text { G2-N } \\
\text { G2-Gr } \\
\text { G3-B } \\
\text { G3-N } \\
\text { G3-Gr }\end{array}$ & $\begin{array}{l}15 \\
6 \\
4 \\
12 \\
6 \\
5 \\
9 \\
5 \\
4\end{array}$ & $\begin{array}{l}51,0 \mathrm{~b} \\
46,2 \mathrm{a} \\
45,8 \mathrm{a} \\
55,8 \mathrm{~d} \\
50,8 \mathrm{~b} \\
50,0 \mathrm{~b} \\
59,3^{\mathrm{e}} \\
54,2 \mathrm{c} \\
53,8 \mathrm{c}\end{array}$ & $\begin{array}{l}1,2 \\
1,6 \\
1,0 \\
1,1 \\
0,7 \\
1,2 \\
1,0 \\
0,8 \\
0,5\end{array}$ & $\begin{array}{l}12 \\
5 \\
4 \\
11 \\
6 \\
4 \\
8 \\
5 \\
4\end{array}$ & $\begin{array}{l}74,1 \mathrm{~b} \\
67,2 \mathrm{a} \\
66,3 \mathrm{a} \\
81,9 \mathrm{c} \\
74,8 \mathrm{~b} \\
73,6 \mathrm{~b} \\
89,2 \mathrm{~d} \\
81,6 \mathrm{c} \\
80,8 \mathrm{c}\end{array}$ & $\begin{array}{l}1,6 \\
2,5 \\
1,5 \\
1,8 \\
0,8 \\
1,7 \\
1,6 \\
1,3 \\
0,5\end{array}$ & $\begin{array}{l}11 \\
5 \\
4 \\
11 \\
5 \\
4 \\
8 \\
5 \\
4\end{array}$ & $\begin{array}{l}91,1 \mathrm{~b} \\
82,0 \mathrm{a} \\
81,0 \mathrm{a} \\
102,5 \mathrm{c} \\
93,5 \mathrm{~b} \\
92,2 \mathrm{~b} \\
113,1 \mathrm{~d} \\
102,1 \mathrm{c} \\
102,0 \mathrm{c}\end{array}$ & $\begin{array}{l}2,0 \\
3,0 \\
2,0 \\
2,3 \\
1,0 \\
2,2 \\
2,1 \\
2,7 \\
1,4\end{array}$ & $\begin{array}{l}11 \\
5 \\
4 \\
11 \\
5 \\
3 \\
8 \\
5 \\
4\end{array}$ & $\begin{array}{l}106,5 \mathrm{~b} \\
96,3 \mathrm{a} \\
95,8 \mathrm{a} \\
121,1 \mathrm{c} \\
108,4 \mathrm{~b} \\
110,3 \mathrm{~b} \\
135,8 \mathrm{~d} \\
123,1 \mathrm{c} \\
123,0 \mathrm{c}\end{array}$ & $\begin{array}{l}2,4 \\
3,2 \\
1,7 \\
2,7 \\
3,3 \\
1,0 \\
2,5 \\
1,0 \\
2,0\end{array}$ & $\begin{array}{l}11 \\
5 \\
4 \\
11 \\
5 \\
3 \\
8 \\
5 \\
4\end{array}$ & $\begin{array}{l}122,5 \mathrm{~b} \\
110,7 \mathrm{a} \\
108,5 \mathrm{a} \\
140,7 \mathrm{~d} \\
128,8 \mathrm{c} \\
124,8 \mathrm{~b} \\
158,8 \mathrm{~d} \\
142,6 \mathrm{~b} \\
143,0 \mathrm{~d}\end{array}$ & $\begin{array}{l}2,8 \\
3,7 \\
3,3 \\
3,2 \\
2,8 \\
2,0 \\
3,1 \\
2,9 \\
1,4\end{array}$ \\
\hline
\end{tabular}

$\mathrm{P} 1$ : à l'éclosion ; P7 : 7 jours après éclosion ; P15: 15 jours après éclosion ; P23 : 23 jours après éclosion ; $\mathrm{P} 30$ : 30 jours après éclosion

Moy $=$ moyenne; E.T.: écart-type; $\mathrm{n}=$ nombre

Les moyennes de la même colonne suivies de la même lettre ne sont pas significativement différentes au seuil de 5\%. 
Tableau 2. Moyenne des moindres carrés et écart-types des gains moyens quotidiens (GMQ en g) des dindonneaux suivant différents stades d'évolution après éclosion.

\begin{tabular}{|c|c|c|c|c|c|c|c|c|c|c|c|c|c|c|c|}
\hline Variables & $\mathbf{n}$ & $\begin{array}{l}\text { GMQ } \\
0-7 \text { j } \\
\text { Moy }\end{array}$ & E.T. & $\mathbf{n}$ & $\begin{array}{l}\text { GMQ } \\
7-15 \mathrm{j} \\
\text { Moy }\end{array}$ & E.T. & $\mathbf{n}$ & $\begin{array}{l}\text { GMQ } \\
15-23 \text { j } \\
\text { Moy }\end{array}$ & E.T. & $\mathrm{n}$ & $\begin{array}{l}\text { GMQ } \\
\text { 23-30 j } \\
\text { Moy }\end{array}$ & E.T. & $\mathbf{n}$ & $\begin{array}{l}\text { GMQ } \\
\text { 0-30 j } \\
\text { Moy }\end{array}$ & E.T. \\
\hline Groupe $(\mathrm{G})$ & & & & & & & & & & & & & & & \\
\hline G1 & 25 & $3,2 \mathrm{a}$ & 0,2 & 21 & $2,0 \mathrm{a}$ & 0,2 & 20 & $1,9 \mathrm{a}$ & 0,1 & 20 & $2,2 \mathrm{a}$ & 0,2 & 20 & $2,3 \mathrm{a}$ & 0,1 \\
\hline G2 & 23 & $3,6 \mathrm{~b}$ & 0,2 & 21 & $2,5 \mathrm{~b}$ & 0,1 & 20 & $2,2 \mathrm{~b}$ & 0,2 & 19 & $2,7 \mathrm{~b}$ & 0,3 & 19 & $2,7 \mathrm{~b}$ & 0,2 \\
\hline G3 & 18 & $4,1 \mathrm{c}$ & 0,2 & 17 & $2,8 \mathrm{c}$ & 0,2 & 17 & $2,8 \mathrm{c}$ & 0,3 & 17 & $3,1 \mathrm{c}$ & 0,4 & 17 & $3,1 \mathrm{c}$ & 0,2 \\
\hline Phénotype & & & & & & & & & & & & & & & \\
\hline Bronzé (B) & 36 & $3,7 \mathrm{~b}$ & 0,4 & 31 & $2,5 \mathrm{~b}$ & 0,4 & 30 & $2,3 \mathrm{a}$ & 0,4 & 30 & $2,7 \mathrm{~b}$ & 0,4 & 30 & $3,8 \mathrm{~b}$ & 0,4 \\
\hline $\mathrm{N}$ (Noir) & 17 & $3,4 a$ & 0,4 & 16 & $2,2 \mathrm{a}$ & 0,3 & 15 & $2,2 \mathrm{a}$ & 0,4 & 15 & $2,5 \mathrm{a}$ & 0,4 & 15 & $2,5 \mathrm{a}$ & 0,3 \\
\hline Gr (Gris) & 13 & $3,4 a$ & 0,4 & 1,2 & $2,3 a b$ & 0,4 & 12 & $2,2 \mathrm{a}$ & 0,4 & 11 & $2,3 a$ & 0,5 & 11 & $2,5 \mathrm{a}$ & 0,4 \\
\hline G*Phénotype & & & & & & & & & & & & & & & \\
\hline G1-B & 15 & $3,3 \mathrm{~b}$ & 0,1 & 12 & $2,1 \mathrm{~b}$ & 0,1 & 11 & $1,9 \mathrm{a}$ & 0,1 & 11 & $2,3 \mathrm{~b}$ & 0,1 & 11 & $2,4 \mathrm{~b}$ & 0,1 \\
\hline G1-N & 6 & $3,0 \mathrm{a}$ & 0,1 & 5 & $1,9 \mathrm{a}$ & 0,1 & 5 & $1,8 \mathrm{e}$ & 0,1 & 5 & $2,0 \mathrm{a}$ & 0,1 & 5 & $2,2 \mathrm{a}$ & 0,1 \\
\hline G1-Gr & 4 & $3,0 \mathrm{a}$ & 0,1 & 4 & $1,9 \mathrm{a}$ & 0,2 & 4 & $1,9 \mathrm{a}$ & 0,1 & 4 & $1,8 \mathrm{a}$ & 0,3 & 4 & $2,1 \mathrm{a}$ & 0,1 \\
\hline G2-B & 12 & $3,8 \mathrm{c}$ & 0,1 & 11 & $2,6 \mathrm{~d}$ & 0,1 & 11 & $2,4 \mathrm{c}$ & 0,1 & 11 & $2,8 \mathrm{c}$ & 0,1 & 11 & $2,8 \mathrm{c}$ & 0,1 \\
\hline G2-N & 6 & $3,3 \mathrm{~b}$ & 0,1 & 6 & $4,2 \mathrm{c}$ & 0,1 & 5 & $2,1 \mathrm{ab}$ & 01 & 5 & $2,6 \mathrm{~cd}$ & 0,2 & 5 & $2,6 \mathrm{bc}$ & 0,1 \\
\hline G2-Gr & 5 & $3,4 \mathrm{~b}$ & 0,1 & 4 & $2,4 \mathrm{c}$ & 0,1 & 4 & $2,0 \mathrm{a}$ & 01 & 3 & $2,3 b$ & 0,3 & 3 & $2,5 \mathrm{~b}$ & 0,1 \\
\hline G3-B & 9 & $4,3 \mathrm{~d}$ & 0,1 & 8 & $3,0 \mathrm{e}$ & 0,1 & 8 & $2,9 \mathrm{e}$ & 01 & 8 & $3,3 \mathrm{e}$ & 0,1 & 8 & $3,3 \mathrm{e}$ & 0,1 \\
\hline G3-N & 5 & $3,9 \mathrm{c}$ & 0,1 & 5 & $2,6 \mathrm{~d}$ & 0,2 & 5 & $2,6 \mathrm{~d}$ & 04 & 5 & $2,8 \mathrm{c}$ & 0,5 & 5 & $3,0 \mathrm{~d}$ & 0,1 \\
\hline G3-Gr & 4 & $3,9 \mathrm{c}$ & 0,1 & 4 & $2,7 \mathrm{~d}$ & 0,2 & 4 & $2,6 \mathrm{~d}$ & 03 & 4 & $2,9 \mathrm{c}$ & 0,3 & 4 & $3,0 \mathrm{~d}$ & 0,1 \\
\hline
\end{tabular}

Moy = moyenne; E.T.: écart-type; $n=$ nombre $; j=$ jours

Les moyennes de la même colonne suivies de la même lettre ne sont pas significativement différentes au seuil de $5 \%$.

Le type d'élevage a eu un effet significatif sur le poids à l'éclosion des dindonneaux. Ainsi, les dindonneaux du groupe 3 sont plus lourds à l'éclosion, suivis de ceux du groupe 2 ; les dindonneaux du groupe 1 sont les plus légers $(\mathrm{p}<0,05)$. Le phénotype a aussi eu un effet significatif sur le paramètre étudié. Les dindonneaux bronzés sont plus lourds $(\mathrm{p}<0,05)$ à l'éclosion que les dindonneaux noirs et gris. Cet avantage constaté au niveau des dindonneaux du groupe 3 et de phénotype bronzé a été maintenu à 7, 15, 23 et 30 jours après éclosion.

A 30 jours après éclosion, l'effet croisé Groupe * Phénotype a montré que les dindonneaux bronzés du groupe 3 ont eu les poids vifs et les GMQ les plus élevés ( $p<0,05)$, suivis par les dindonneaux gris et noirs du groupe 3 et bronzés du groupe 2 . Les dindonneaux noirs du groupe 2 et bronzés du groupe 1 ont occupé respectivement la $3^{\mathrm{e}}$ et la $4^{\mathrm{e}}$ place. Enfin, les dindonneaux noirs et gris du groupe 1 ont eu les poids vifs et GMQ les plus bas $(\mathrm{p}<0,05)$.

\section{Evolution du poids vif des dindons}

Les poids vifs des dindons des deux sexes à 3 et 6 mois, des dindes (PFA) et dindons (PMA) adultes sont consignés dans le tableau 3. 
Tableau 3. Moyenne des moindres carrés et écart-types des poids $(\mathrm{kg})$ des dindons à $3(\mathrm{P} 3)$, 6 mois (PF6 et PM6) et des dindons adultes (PFA et PMA).

\begin{tabular}{|c|c|c|c|c|c|c|c|c|c|c|c|c|c|c|c|}
\hline Variables & n & $\begin{array}{l}\text { P3 } \\
\text { Moyen } \\
\text { ne } \\
\end{array}$ & E.T. & $\mathbf{n}$ & $\begin{array}{l}\text { PF6 } \\
\text { Moyen } \\
\text { ne } \\
\end{array}$ & E.T. & $\mathbf{n}$ & $\begin{array}{l}\text { PM6 } \\
\text { Moyen } \\
\text { ne } \\
\end{array}$ & E.T. & $\mathbf{n}$ & $\begin{array}{l}\text { PFA } \\
\text { Moyen } \\
\text { ne }\end{array}$ & E.T. & $\mathbf{n}$ & $\begin{array}{l}\text { PMA } \\
\text { Moyen } \\
\text { ne } \\
\end{array}$ & E.T. \\
\hline $\begin{array}{l}\text { GROUPE (G) } \\
\text { G1 } \\
\text { G2 } \\
\text { G3 } \\
\end{array}$ & $\begin{array}{l}21 \\
17 \\
15 \\
\end{array}$ & $\begin{array}{l}0,78 \mathrm{a} \\
0,90 \mathrm{~b} \\
1,19 \mathrm{c} \\
\end{array}$ & $\begin{array}{l}0,08 \\
0,08 \\
0,26 \\
\end{array}$ & $\begin{array}{l}33 \\
29 \\
20 \\
\end{array}$ & $\begin{array}{l}2,66 \mathrm{a} \\
3,03 \mathrm{ab} \\
3,32 \mathrm{~b}\end{array}$ & $\begin{array}{l}0,80 \\
0,93 \\
1,3 \\
\end{array}$ & $\begin{array}{l}14 \\
13 \\
12 \\
\end{array}$ & $\begin{array}{l}2,88 \mathrm{a} \\
2,90 \mathrm{a} \\
3,24 \mathrm{a} \\
\end{array}$ & $\begin{array}{l}1,2 \\
0,80 \\
0,17 \\
\end{array}$ & $\begin{array}{l}32 \\
29 \\
20 \\
\end{array}$ & $\begin{array}{l}2,12 \mathrm{a} \\
2,97 \mathrm{~b} \\
3,07 \mathrm{~b}\end{array}$ & $\begin{array}{l}1,24 \\
0,94 \\
1,26 \\
\end{array}$ & $\begin{array}{l}13 \\
12 \\
12 \\
\end{array}$ & $\begin{array}{l}5,15 \mathrm{a} \\
7,17 \mathrm{~b} \\
8,42 \mathrm{c} \\
\end{array}$ & $\begin{array}{l}1,46 \\
1,27 \\
1,08 \\
\end{array}$ \\
\hline $\begin{array}{l}\text { PHENOTYPE } \\
\text { B (Bronzé) } \\
\text { N (Noir) } \\
\text { Gr (Gris) } \\
\end{array}$ & $\begin{array}{l}26 \\
13 \\
14 \\
\end{array}$ & $\begin{array}{l}1,03 \mathrm{~b} \\
0,83 \mathrm{a} \\
0,86 \mathrm{a} \\
\end{array}$ & $\begin{array}{l}0,28 \\
0,11 \\
0,11 \\
\end{array}$ & $\begin{array}{l}47 \\
19 \\
16 \\
\end{array}$ & $\begin{array}{l}3,30 \mathrm{~b} \\
2,53 \mathrm{a} \\
2,43 \mathrm{a} \\
\end{array}$ & $\begin{array}{l}1,1 \\
0,72 \\
0,64 \\
\end{array}$ & $\begin{array}{l}18 \\
11 \\
10 \\
\end{array}$ & $\begin{array}{l}3,17 \mathrm{a} \\
2,85 \mathrm{a} \\
2,84 \mathrm{a} \\
\end{array}$ & $\begin{array}{l}1,02 \\
0,30 \\
0,32 \\
\end{array}$ & $\begin{array}{l}46 \\
19 \\
16 \\
\end{array}$ & $\begin{array}{l}2,51 \mathrm{a} \\
2,71 \mathrm{a} \\
3,04 \mathrm{a} \\
\end{array}$ & $\begin{array}{l}1,3 \\
1,20 \\
1,21 \\
\end{array}$ & $\begin{array}{l}18 \\
10 \\
9 \\
\end{array}$ & $\begin{array}{l}6,78 \mathrm{a} \\
6,8 \mathrm{a} \\
7,11 \mathrm{a} \\
\end{array}$ & $\begin{array}{l}2,26 \\
1,39 \\
1,54 \\
\end{array}$ \\
\hline $\begin{array}{l}\text { G*PHENOTY } \\
\text { PE } \\
\text { G1-B } \\
\text { G1-N } \\
\text { G1-Gr } \\
\text { G2-B } \\
\text { G2-N } \\
\text { G2-Gr } \\
\text { G3-B } \\
\text { G3-N } \\
\text { G3-Gr }\end{array}$ & $\begin{array}{l}13 \\
4 \\
4 \\
7 \\
5 \\
5 \\
6 \\
4 \\
5\end{array}$ & $\begin{array}{c}0,83 \mathrm{~b} \\
0,72 \mathrm{a} \\
0,71 \mathrm{a} \\
1,00 \mathrm{c} \\
0,85 \mathrm{~b} \\
0,83 \mathrm{~b} \\
1,50 \mathrm{~d} \\
0,96 \mathrm{c} \\
0,99 \mathrm{c}\end{array}$ & $\begin{array}{l}0,08 \\
0,02 \\
0,02 \\
0,02 \\
0,01 \\
0,02 \\
0,03 \\
0,03 \\
0,02\end{array}$ & $\begin{array}{l}20 \\
9 \\
4 \\
17 \\
5 \\
7 \\
10 \\
5 \\
5\end{array}$ & $\begin{array}{l}2,67 \mathrm{a} \\
2,74 \mathrm{a} \\
2,45 \mathrm{a} \\
3,49 \mathrm{~b} \\
2,19 \mathrm{a} \\
2,50 \mathrm{a} \\
4,23 \mathrm{c} \\
2,50 \mathrm{a} \\
2,31 \mathrm{a}\end{array}$ & $\begin{array}{l}0,77 \\
0,90 \\
0,95 \\
0,84 \\
0,45 \\
0,74 \\
1,30 \\
0,51 \\
0,06\end{array}$ & $\begin{array}{l}9 \\
3 \\
2 \\
5 \\
4 \\
4 \\
4 \\
4 \\
4\end{array}$ & $\begin{array}{l}3,11 \mathrm{~b} \\
2,55 \mathrm{a} \\
2,36 \mathrm{a} \\
3,64 \mathrm{c} \\
2,79 \mathrm{a} \\
2,78 \mathrm{a} \\
4,48 \mathrm{~d} \\
3,13 \mathrm{~b} \\
3,14 \mathrm{~b}\end{array}$ & $\begin{array}{l}1,46 \\
0,39 \\
0,09 \\
0,07 \\
0,04 \\
0,15 \\
0,06 \\
0,08 \\
0,03\end{array}$ & $\begin{array}{l}19 \\
9 \\
4 \\
17 \\
5 \\
7 \\
10 \\
5 \\
5\end{array}$ & $\begin{array}{l}2,97 \mathrm{a} \\
2,34 \mathrm{a} \\
2,39 \mathrm{a} \\
2,82 \mathrm{a} \\
3,47 \mathrm{a} \\
2,80 \mathrm{a} \\
3,00 \mathrm{a} \\
3,26 \mathrm{a} \\
3,02 \mathrm{a}\end{array}$ & $\begin{array}{l}0,76 \\
0,58 \\
1,01 \\
0,89 \\
1,24 \\
0,45 \\
1,80 \\
0,73 \\
1,2\end{array}$ & $\begin{array}{l}9 \\
2 \\
2 \\
5 \\
4 \\
3 \\
4 \\
4 \\
4\end{array}$ & $\begin{array}{l}4,89 \mathrm{a} \\
6,50 \mathrm{a} \\
5,00 \mathrm{a} \\
8,4 \mathrm{c} \\
6,00 \mathrm{a} \\
6,67 \mathrm{~b} \\
9,01 \mathrm{c} \\
7,75 \mathrm{~b} \\
8,50 \mathrm{c}\end{array}$ & $\begin{array}{l}1,61 \\
0,71 \\
0,05 \\
0,55 \\
0,82 \\
0,58 \\
0,07 \\
1,70 \\
0,58\end{array}$ \\
\hline
\end{tabular}

Moy = moyenne; E.T.: écart-type; $\mathrm{n}=$ nombre

P3 : poids à 3 mois ; PF6 : poids des femelles à 6 mois ; PM6 : poids des males à 6 mois ; PFA : poids des femelles adultes ; PMA : poids des males adultes

Les moyennes de la même colonne suivies de la même lettre ne sont pas significativement différentes au seuil de $5 \%$.

A 3 mois après éclosion, l'effet groupe a été significatif. Les dindonneaux du groupe 3 sont les plus lourds ( $<<0,05)$, suivis par ceux du groupe 2 , qui sont différents $(\mathrm{p}<0,05)$ des dindonneaux du groupe 1 . Le phénotype des dindonneaux a eu un effet significatif sur leur poids vif à 3 mois après éclosion. Les bronzés ont eu un poids vif supérieur $(\mathrm{p}<0,05)$ à ceux des noirs et gris. Ces différentes observations ont été confirmées par l'effet conjugué Groupe * Phénotype sur les poids vifs des animaux à 3 mois.

A 6 mois après éclosion, on constate que les mâles et les femelles ont les mêmes poids vifs. L'effet du groupe est significatif chez les femelles et ne l'est pas chez les mâles : les femelles du groupe 3 ont eu un poids vif supérieur $(\mathrm{p}<0,05)$. L'effet du phénotype existe chez les femelles (les bronzées sont plus lourdes) et pas chez les males. La croissance pondérale des dindonneaux mâles s'est uniformisée pendant la tranche d'âge de 3 à 6 mois ; alors que celle des femelles est demeurée sous l'influence du groupe et du phénotype.

Le poids vif des mâles a pratiquement doublé entre 6 mois et l'âge adulte ; alors que le poids vif des femelles n'a pratiquement pas varié entre 6 mois et l'âge adulte. Cependant, le poids vif des dindes adultes a été significativement influencé par le groupe et non par le phénotype et par l'effet conjugué des deux facteurs.

Le groupe a eu un effet significatif sur le poids vif des dindons adultes : 
les dindons du groupe 3 sont plus lourds $(\mathrm{p}<0,05)$ que ceux du $2^{\mathrm{e}}$ groupe et ceux du groupe 1 sont les plus légers $(p<0,05)$. Le phénotype n'a pas eu d'effet sur le poids vif des dindons, alors que l'effet conjugué des deux facteurs a été au contraire significatif. Ainsi, dindons adultes bronzés des groupes 2 et 3 et les gris du groupe 3 sont les plus lourds $(p<0,05)$ et les bronzés et gris du groupe 1 , les plus légers $(p<0,05)$.

\section{Performances de ponte et de reproduction des dindes}

Les résultats de la performance de ponte et de la reproduction des dindes sont consignés dans le tableau 8. Les dindes du groupe 3 ont eu de meilleures $(\mathrm{p}<0,05)$ performances de ponte et de reproduction que celles des deux autres groupes. Les dindes du $2^{\mathrm{e}}$ groupe ont eu des performances intermédiaires $(\mathrm{p}<0,05)$ entre celles des groupes 3 et 1 .

Ainsi, les dindes du groupe 3 ont pondu plus tôt, beaucoup plus d'œufs plus lourds et plus de fois dans l'année que les dindes des 2 autres lots. Elles n'ont été égalées par les dindes du $2^{\mathrm{e}}$ groupe que par le nombre de couvées par an. Ces bonnes performances de ponte ont conduit de meilleures performances de reproduction et à un taux d'éclosion, supérieur $(\mathrm{p}<0,05)$ de 48,3\% et $18,7 \%$ respectivement par rapport à ceux des dindes des groupes 1 et 2 (tableau 4).

Tableau 4 : Résultats de la performance de ponte et de reproduction des dindes selon le type d'élevage (moyenne \pm écart-type)

\begin{tabular}{|l|l|l|l|}
\hline Paramètres & Groupe 1 & Groupe 2 & Groupe 3 \\
\hline Age de première ponte (mois) & $8,41 \mathrm{a} \pm 1,26$ & $7,5 \mathrm{~b} \pm 0,92$ & $6,54 \mathrm{c} \pm 0,76$ \\
\hline $\begin{array}{l}\text { Nombre d'œufs pondus par } \\
\text { couvée }\end{array}$ & $9,3 \mathrm{a} \pm 1,2$ & $10,1 \mathrm{~b} \pm 0,9$ & $11,0 \mathrm{c} \pm 0,9$ \\
\hline Poids des œufs (g) & $60,4 \mathrm{a} \pm 3,1$ & $62,7 \mathrm{~b} \pm 2,9$ & $67,9 \mathrm{c} \pm 3,2$ \\
\hline Nombre de couvée par an & $2,6 \mathrm{a} \pm 0,5$ & $3,0 \mathrm{~b} \pm 0,7$ & $3,1 \mathrm{c} \pm 0,6$ \\
\hline Nombre d'œufs pondus par an & $24,1 \mathrm{a} \pm 5,6$ & $30,4 \mathrm{~b} \pm 7,0$ & $33,4 \mathrm{c} \pm 5,2$ \\
\hline Nombre d'œufs incubés par an & $19,0 \mathrm{a} \pm 3,7$ & $25,1 \mathrm{~b} \pm 5,3$ & $28,8 \mathrm{c} \pm 5,0$ \\
\hline Nombre d'œufs éclos par an & $10,5 \mathrm{a} \pm 2,1$ & $16,5 \mathrm{~b} \pm 3,4$ & $20,3 \mathrm{c} \pm 3,6$ \\
\hline Taux d'éclosion \% & $55,4 \mathrm{a} \pm 1,3$ & $65,7 \mathrm{~b} \pm 1,0$ & $70,5 \mathrm{c} \pm 0,9$ \\
\hline
\end{tabular}

Moy = moyenne; E.T.: écart-type; $n=$ nombre

Les moyennes de la même colonne suivies de la même lettre ne sont pas significativement différentes au seuil de 5\%.

Le type d'élevage a donc eu un effet significatif sur les performances de ponte et de reproduction des dindes.

\section{Discussion}

L'évolution des poids des dindons a montré que les éleveurs du groupe 3 ont des animaux plus lourds. Ces éleveurs, certainement entretiennent mieux 
leurs animaux. Ils ont un meilleur suivi sanitaire et pratiquent la complémentation des dindonneaux avec des termites, des asticots, les résidus de fabrication de la bière locale et de l'akassa, certains achètent même la farine de poisson (Dèdéhou et al., 2018). Dans le groupe 2, les animaux bénéficient de moins de soins et d'aliments et dans le groupe 1, ils sont pratiquement abandonnés (Dèdéhou et al., 2018). Ils doivent complémenter leur ration en consommant insectes, vers, verdure, graines diverses. L'abreuvement se fait le plus souvent dans les mares, flaques d'eau ou vieux ustensiles abandonnés dans la cour des concessions, ce qui constitue des risques de parasitisme (Dèdéhou et al., 2018). Les poids vifs des dindes des différents groupes sont assez proches de ceux de Halbouche et al. (2010) qui ont rapporté des poids vifs variant entre 2,7 et $2,8 \mathrm{~kg}$ pour des dindes adultes dans des élevages familiaux.

A 3 mois après éclosion, le poids des dindonneaux variant entre 0,78 à $1,19 \mathrm{~kg}$, et le phénotype ont eu des effets significativement différents. Halbouche et al. (2010) ont rapporté pour des dindonneaux de même âge un poids vif de $1,394 \mathrm{~kg}$ (pour les mâles) et 1,433 kg (pour les femelles), soulignant qu'il n'y a aucun dimorphisme sexuel exprimé durant cette phase d'élevage. Ces auteurs ont aussi montré que le dindonneau local manifeste une croissance lente et des performances faibles malgré le rationnement alimentaire et sa distribution à volonté, ce qui laisse supposer un déterminisme génétique du petit format de l'animal.

L'effet des deux facteurs que sont le groupe et le phénotype s'est maintenu sur le poids vif des mâles adultes. Chez les femelles adultes par contre, le poids vif n'a pas été significativement différent en relation avec le phénotype. Ce résultat est en accord avec ceux obtenus par Halbouche et al. (2010). La croissance des femelles s'est arrêtée à partir de 6 mois d'âge certainement à cause du démarrage de la reproduction. En effet la ponte, la couvaison et l'élevage des petits sont des activités qui épuisent les dindes et ne leur permettent pas d'accroître leur poids vif surtout quand elles sont mal nourries et mal entretenues. Selon Koundou (1994), le poids vif des dindes serait passé de $3 \mathrm{~kg}$ en début de couvaison à 2,5 kg en fín de couvaison. Elles auraient ainsi perdu $500 \mathrm{~g}$ de poids vif en 28 jours de couvaison soit près de 18 g par jour. Pendant ce temps, les dindons poursuivent leur croissance et c'est en ce moment que l'écart se creuserait entre mâles et femelles.

Les performances de ponte et de reproduction des dindes du groupe 3 ont été meilleures par rapport à celles des deux autres groupes. Ces résultats montrent que l'amélioration des conditions d'élevage donne de bons résultats en élevage des dindons. Dans notre étude, l'âge à la première ponte a varié de 6,5 mois à 8,4 mois et le nombre d'œufs par couvée de 9,3 à 11 . Ces résultats sont similaires à ceux rapportés par Salifou (1977) et Koundou (1994) où l'âge à la ponte a varié de 6 à 8 mois et chaque dinde a pondu 9 à 10 œufs par couvée 
en l'espace de 3 semaines environ. Le poids des œufs obtenu dans cette étude $(60,4 \mathrm{~g}$ à $67,9 \mathrm{~g})$ est inférieur à ceux obtenus par Boudina (2002) et Halbouche et al. (2010) qui ont rapporté des poids d'œufs de dinde locale variant entre $78.5 \mathrm{~g}$ et $83,6 \mathrm{~g}$. la différence observée entre le nombre d'œufs pondus et le nombre d'œufs incubés serait due aux pertes par cassure des œufs et par prédation, qui serait facilité par l'état rudimentaire des bâtiments d'élevage (Dèdéhou et al., 2018). Les taux d'éclosion ont varié entre 55,4\% et 70,5\% suivant les groupes et le groupe $1 \mathrm{a}$ eu le plus faible taux d'éclosion. Ceci se justifierait par le fait que certains élevages de ce groupe ne possèdent pas de dindons reproducteurs et empruntent les géniteurs des voisins pour accoupler leurs dindes (Dèdéhou et al., 2018). Du fait du mode d'élevage en liberté, théoriquement quelques dindons (mâles) dans le village suffisent pour que même les propriétaires de dindes sans géniteurs aient des éclosions plus ou moins régulières. Mais, dans la pratique, le taux d'éclosion dans les élevages sans géniteurs est plus faible. Toutefois, l'absence d'une alimentation adéquate des géniteurs peut entraîner la baisse de la qualité des semences. Les taux d'éclosion obtenus dans cette étude sont inférieurs 79\% rapporté par Halbouche et al. (2010) et supérieur à 55\% rapporté par Boudina (2002).

Le poids vif moyen du dindonneau à la naissance a varié significativement entre groupes et entre phénotype. Ces résultats concordent avec ceux de Halbouche et al. (2010) et Boudina (2002) mais pour Halbouche et al. (2010), le poids à la naissance du dindonneau n'est pas influencé par le phénotype.

Dans l'ensemble, ces performances de reproduction demeurent faibles et variables lorsqu'elles sont comparées à celles de dindes menées en systèmes intensifs ou fermiers. Dans ces systèmes, le total des pertes enregistrées dues à l'infertilité des œufs et à la mortalité embryonnaire ne dépasse pas $20 \%$ (Agreste 2006, Molette et Guerin 2010). Aussi, Camacho-Escobar et al. (2008) rapportent un taux d'éclosion moyen de $76 \%$ et un taux d'éclosivité de $85 \%$ dans des élevages mexicains fermiers.

\section{Conclusion}

Cette étude a montré beaucoup de variations en termes de performances de croissance pondérale, de ponte et de reproduction des dindons selon les différents groupes. Il ressort de cette étude que l'élevage du dindon local en milieu paysan connaît des insuffisances du point de vue croissance, reproduction, système de production et environnement. La réussite de cet élevage passe par la mise en place d'un habitat adéquat, une alimentation améliorée et par un assainissement de l'environnement. Ceci nécessite la mise en place d'une formation plus efficace des acteurs de la filière en vue du renforcement des capacités des aviculteurs. 


\section{References :}

1. Agreste. (2006). Données de cadrage aviculture (poulets de chair, dinde). Avicadre $\mathrm{N}^{\circ} 3,19$ Avril 2006, 5 pp.

2. Alekseev, F.F. (2005). Turkey-prospect poultry meat. Ptica $i$ Pticeprodukty, 5: 12-15.

3. Ascherio, A., Willett, W.C., Rimm, E.B., Giovannucci, E.L., Stampfer, M.J. (1994). Dietary iron intake and risk of coronary disease among men. Circulation, 89 : 969-974.

4. Baggio, S.R., Vicente, E., Bragagnolo, N. (2002). Cholesterol oxides, cholesterol, total lipid and fatty acid composition in turkey meat. Journal of Agricultural and Food Chemistry, 50 : 5981-5986.

5. Banque Centrale des Etats de l'Afrique de l'Ouest (BCEAO). (2017). Annuaire statistique, pp. 3- 7.

6. Boudina, H. (2002). Bilan des performances de la population locale de dindes élevées à la station de Baba Ali. ITELV, Algérie, Document ronéotypé, pp 10.

7. Camacho-Escobar, M.A., Hernandez-Sanchez, V., Ramirez-Cancino, L., Sanchez-Bernal, E.I., Arroyo-Ledezma, J. (2008). Characterization of backyard guajolotes (Meleagris gallopavo gallopavo) in tropical zones of Mexico. Livestock Research for Rural Development, 20(4), 18.

8. Fanou, U. (2006). Première évaluation de la structure et de l'importance du secteur avicole commercial et familial en Afrique de l'Ouest - Cas du Bénin (Enquête CPVBP, 2006) Rapport FAO, pp. 332.

9. Halbouche, M., Mouats, A., Belhadi, A., Homrani, A., Didi, M. (2010). Ponte, reproduction et développement sexuel chez la dinde domestique (Meleagris Gallopavo) en Algérie: Influence du phénotype et du niveau protéique des aliments. European Journal of Scientific Research 47(3) : 473-483.

10. Koundou, I.B. (1994). Quelques aspects de l'élevage du dindon en République du Bénin. Thèse d'Ingénieur Agronome, Faculté des Sciences Agronomiques, Université d'Abomey-Calavi, AbomeyCalavi, pp 166.

11. Lhoste, P., Dollé, V., Rousseau, J., Soltner, D. (1993). Manuel de zootechnie des régions chaudes : les systèmes d'élevage. Collection précis d'élevage, Ministère de la coopération Française, pp 281.

12. Ministère de l'Agriculture, de l'Elevage et de la Pêche (MAEP). (2014). Rapport de Performance du Secteur Agricole, Gestion 2013, pp 3- 47.

13. Molette C, Guerin JL. (2010). Accouvage: développement embryonnaire et incubation. Ecole Nationale Vétérinaire de Toulouse, Document Powerpoint, in www.avicampus.fr, 49 p. 
14. Ribarski, S., Lalev, M., Oblakova, M. (2001). Phisico-chemical characteristics and micromorphological feature of turkey skeleton musculature. Journal of Animal Science, 2 : 106-109.

15. Salifou, K. (1977). Elevage du dindon dans la province de l'Atacora: Possibilités d'amélioration. Mémoire DEAT, Lycée agricole Medji de Sekou, pp 56.

16. SAS. (1989). SASSTAT : User's guide (ressource électronique)version 6- 4ème éd.- New York : Cary, SAS inst.

17. Sonaiya, E.B., Swan, S.E.J. (2004). Production en aviculture familiale, Manuel FAO de Production et Santé Animales. Ed. FAO, Rome, IBS 92-5-205082-5, pp136. 